\title{
Trivium
}

Revue franco-allemande de sciences humaines et sociales - Deutsch-französische Zeitschrift für Geistesund Sozialwissenschaften

$21 \mid 2016$

Autorégulation régulée. Analyses historiques de structures de régulation hybrides

\section{Frankreich nach 1789 - eine unmögliche Zivilgesellschaft?}

\section{Claire Lemercier}

Traducteur : Bettina Engels et Michael Adrian

\section{OpenEdition \\ Journals}

Édition électronique

URL : http://journals.openedition.org/trivium/5237

ISSN : 1963-1820

Éditeur

Les éditions de la Maison des sciences de l'Homme

\section{Référence électronique}

Claire Lemercier, «Frankreich nach 1789 - eine unmögliche Zivilgesellschaft? », Trivium [Online], 21 |

2016, online erschienen am 10 Mai 2016, abgerufen am 08 September 2020. URL : http://

journals.openedition.org/trivium/5237

Ce document a été généré automatiquement le 8 septembre 2020

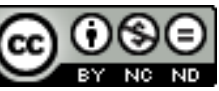

Les contenus des la revue Trivium sont mis à disposition selon les termes de la Licence Creative Commons Attribution - Pas d'Utilisation Commerciale - Pas de Modification 4.0 International. 


\title{
Frankreich nach 1789 - eine unmögliche Zivilgesellschaft?
}

\author{
Claire Lemercier
}

Traduction : Bettina Engels et Michael Adrian

\section{NOTE DE L'ÉDITEUR}

Wir danken Claire Lemercier für die freundliche Genehmigung, diesen Artikel in deutscher Übersetzung zu publizieren.

Nous remercions Claire Lemercier de nous avoir accordé l'autorisation de traduire ce texte pour le présent numéro.

Über:

Steven L. Kaplan / Philippe Minard (Hg.): La France, malade du corporatisme? XVIII ${ }^{e}-X X^{e}$ siècles,

Paris: Belin 2004, 556 S., $26 €$. Pierre Rosanvallon: Le modèle politique français. $\mathrm{La}$ société civile contre le jacobinisme de 1789 à nos jours,

Paris: Seuil 2004, 460 S., $25 €$.

Ab sofort sollte kein Historiker mehr leichtfertig die Begriffe "Jakobinismus" und "Korporatismus« verwenden, insbesondere nicht, um einen vermeintlichen "französischen Sonderweg" zu charakterisieren. Denn ein Jahrzehnt der Erkundung jenes ungewissen Ortes, den kollektive Organisationen jeglicher Art - »intermediäre Körperschaften«? - in Frankreich einnehmen, fand durch zwei wichtige, einander ergänzende Neuerscheinungen seinen krönenden Abschluss. Diese Körperschaften werden dort im Hinblick auf ihre ökonomischen, sozialen und politischen Funktionen sowie in ihrem Verhältnis zum Staat untersucht; einem Verhältnis, das oft schwierig ist oder zumindest lieber beschwiegen wird, seit das berühmte Gesetz Le Chapelier ein grundsätzliches Verbot sämtlicher Gruppierungen vom Typ einer Vereinigung oder einer Gewerkschaft verfügte. ${ }^{1}$ 
2 Genauso wenig kann man sich noch an die alte als "tocquevillesch" apostrophierte Glaubenslehre halten, die immer abwechselnd den sich in alles einmischenden Staat und das allmächtige Individuum anprangert. Die Existenz und die Vitalität von Gewerkschaften, Verbänden und Organisationen anderer Art waren schon vor ihrer Duldung und späteren rechtlichen Anerkennung, ja Indienstnahme durch den Staat nicht zu übersehen. Doch geht es nicht darum, den nachhaltigen Einfluss zu leugnen, den bis heute jener Kult um das einer Organisation der Partikularinteressen angeblich entgegenstehende Gemeininteresse besitzt. Dieser zunehmend modifizierte, aber immer noch dominante »Diskurs der Allgemeinheit« belastet die Praktiken auch dann, wenn sie nicht unbedingt dem Buchstaben des Gesetzes folgen. So schreiben Steven L. Kaplan und Philippe Minard: "Das Gesetz Le Chapelier hat die Entwicklung der Gewerkschaftsbewegung nicht nur verzögert, es hat über lange Zeit auch ihren Charakter beeinflusst«; und es ist gerade diese gleichzeitige Berücksichtigung der Diskurse und der Praktiken, die den hier erörterten Werken ihre Kraft verleiht. ${ }^{2}$ Wie Pierre Rosanvallon in der Einleitung seines Buches betont, gewinnen wir ein sehr unterschiedliches Bild von Frankreich, je nachdem ob wir die Ideengeschichte oder die Sozialgeschichte seiner kollektiven Organisationen betrachten. Die Absicht, diese verschiedenen Bilder einander gegenüberzustellen und in ihrem Zusammenhang zu verstehen, wurde in beiden hier zu besprechenden Büchern weitgehend eingelöst.

3 Auch nachdem zunächst die Gewerkschaften und später die Vereinigungen offiziell anerkannt worden waren, blieb die Bestimmung ihres rechtlichen Status, ihrer Rolle im Verhältnis zum Staat und ihrer Beziehung zur Politik ausgesprochen schwierig. Die Zäsuren von 1884 und 1901 sehen sich durch diesen Umstand weitgehend relativiert: Beide Bücher heben die Entwicklungen hervor, die im Laufe des 19. und des 20. Jahrhunderts stattgefunden haben, verweisen aber auch darauf, dass der Status derartiger Zusammenschlüsse - in den Diskursen wie im Recht - weiterhin mit Unbehagen beäugt wurde. Wie auf je eigene Weise Pierre Rosanvallon und Jean-Pierre Hirsch in einem scharfsinnigen Beitrag des Sammelbands ${ }^{3}$ unterstreichen, führte die Durchsetzung der "Vereinigungsfreiheit« nicht wirklich zu einer Anerkennung der Vereinigung als einer Institution, die über eigene Mittel verfügt, sich selbst verwaltet und gar zur Bildung eines Gemeininteresses beiträgt. "Gewiss wird das Vereinigungsrecht Anfang der 1880er Jahre lautstark als Grundrecht verkündet. Was aber die Institutionen betrifft, die es hervorbringen kann, bleibt es auch weiterhin Gegenstand stets unterschwelliger Unsicherheiten«, resümiert Rosanvallon (S. 321). Trotz dieser Unentschiedenheit der politischen Diskurse, die zu einer Reihe juristischer Einschränkungen führt, vermehren sich die kollektiven Organisationen stark, und die Gewerkschaften erfahren echte institutionelle Anerkennung: Solchen komplexen Diskrepanzen zwischen Diskurs und Praxis versuchen die hier behandelten Werke auf den Grund zu gehen.

4 Trotz ihrer zweifellos provokativ gemeinten, der Subtilität ihrer Thesen aber nicht recht angemessenen Titel zeichnen sie die Geschichte der "Zivilgesellschaft» à la française äußerst differenziert nach - auch wenn es beide Werke vermeiden, diesen stark aufgeladenen Terminus zu verwenden. ${ }^{4}$ In einem Land, in dem der Begriff des "Korporatismus«, sofern er sich nicht auf extrem vage organisatorische Vorhaben bezog, eigentlich immer nur abwertend gebraucht wurde, verfügt die »Zivilgesellschaft« im politischen Diskurs zweifellos nicht über dieselbe Legitimität wie andernorts. Sie wird entweder ignoriert oder als neue Errungenschaft beschworen - 
man denke nur an die "aus der Zivilgesellschaft hervorgegangenen Minister" der letzten Jahre. In der Praxis aber gibt es Regulierungs-, Repräsentations- und Informationsbedürfnisse. Und diese Bedürfnisse werden nicht zuletzt von Organisationsformen befriedigt, die die Zentralverwaltung toleriert und sich häufig auch zu Diensten macht: Nicht immer sind sie "gegen den Staat« gerichtet, ganz im Gegenteil. Beide hier analysierten Werke möchten die Bedeutung der Existenz dieses Typs von Organisation betonen und seine Geschichte nachzeichnen; zugleich gehen sie den Gründen für dessen Delegitimierung im politischen Diskurs nach. Diese Delegitimierung mag im Übrigen auch ein Grund dafür sein, warum die Historiker diese Organisationen so lange übersehen konnten. Die $\mathrm{zu}$ neuem Leben erweckte Historiographie der "Zivilgesellschaft« eröffnet über ihre Bedeutung für die politische Geschichte und die Wirtschaftsgeschichte hinaus nun auch Wege für eine fruchtbare Erforschung der Geschichte der Berufsstände und des Staates.

\section{"Jakobinismus « und "Korporatismus« - zwischen Worten und Praktiken}

5 Die beiden Bücher beleuchten die ihnen gemeinsame allgemeine Fragestellung aus einem je anderen Blickwinkel. Ungeachtet ihrer Titel ist zunächst die Chronologie nicht die gleiche: Während Pierre Rosanvallon das 19. Jahrhundert und insbesondere die Schlüsselperiode zwischen 1864 und 1901 detailliert behandelt, bieten die Autorinnen und Autoren von La France, malade du corporatisme?, auch wenn manche Beiträge andere Epochen berühren, insgesamt mehr Aufschluss über die Revolutionsepoche im weiteren Sinne (von 1776 bis etwa 1840) sowie über die Zwischenkriegszeit und das VichyRegime. Überraschenderweise ist in beiden Büchern nur sehr wenig von der Zweiten Republik die Rede. Rosanvallon geht sogar so weit, ihre Bedeutung für die historische Entwicklung der Positionen zur Vereinigungs- und Gewerkschaftsfrage zu relativieren, was man durchaus in Frage stellen könnte.

In thematischer und methodischer Hinsicht überrascht es hingegen wenig, dass man es hier mit der - von Rosanvallon so sehr geschätzten - "Begriffsgeschichte des Politischen« zu tun bekommt, die auch weiterhin auf ihre Anhänger und Kritiker zählen dürfte. ${ }^{5}$ Doch sollte man in diesem Zusammenhang darauf hinweisen, dass es sich hier selbstverständlich nicht um eine abgehobene oder ahistorische »Ideengeschichte« handelt. Denn auch wenn sich Rosanvallon vor allem mit dem Denken der Regierenden und der Republikaner auseinandersetzt, so bezieht er es doch stets auf die praktischen Zwänge des Regierungsgeschäfts, die realen Umstände der spontanen Bildung kollektiver Organisationen oder auf größere Elemente des ökonomischen und sozialen Kontextes. Auch wenn die vorliegende Monographie, die das wichtige theoretische Problem einer Organisation der Beziehungen zwischen Staat und Zivilgesellschaft aufwirft, als krönender Abschluss einer Reihe von Arbeiten des Autors $\mathrm{zu}$ den Aporien der Demokratie betrachtet werden kann, ${ }^{6}$ so ist sie doch zugleich fest in der Chronologie des 19. Jahrhunderts verankert, für das Rosanvallon wirklich ein Spezialist ist. Man findet bei ihm Verweise auf gedruckte Quellen, die andere oft übersehen. $\mathrm{Zu}$ bedauern ist allerdings, dass er sich kaum auf jüngere Literatur bezieht, auch fehlt leider eine allgemeine Bibliographie. Positiv sei darauf verwiesen, dass das Buch über ein sehr detailliertes und praktisches Inhaltsverzeichnis und Register verfügt. 
7 Mit seiner Gesamtbibliographie für alle Beiträge und seiner ausgesprochen klugen Einleitung hat der von Steven L. Kaplan und Philippe Minard herausgegebene Band allerdings zwei wesentliche Pluspunkte zu verzeichnen: Diese wiegen zum Teil die Unzulänglichkeiten und Disparatheiten auf, die Konferenzvorträge - die vorliegenden wurden 1999 in Ithaca gehalten - oft selbst dann noch auszeichnen, wenn sie eingehend überarbeitet wurden. Der erste und der dritte Teil des Sammelbands, in denen es um Korporationen, um ihre Abschaffung, um die Debatten über ihre Wiedereinrichtung und schließlich um die »unsichere Organisation der kollektiven Arbeitsbeziehungen« geht, knüpfen an die Arbeiten Rosanvallons an. Auch wenn der dritte, auf das 20. Jahrhundert beschränkte Teil Institutionen wie dem Nationalen Wirtschaftsrat, Tarifverträgen und Schlichtungen einen gewissen Raum zubilligt, befasst er sich doch im Wesentlichen mit korporatistischen Doktrinen und Vorhaben. Mit den »beruflichen Identitäten und Solidaritäten « und ihrem Verhältnis zu Organisationsformen und kollektivem Handeln widmet sich der zweite Teil des Werkes einem ergänzenden Aspekt, der hier in sechs Studien zu bestimmten sozialen Gruppen beleuchtet wird. Insgesamt kann man konstatieren, dass der Band, der eine "Sozialgeschichte des Politischen« (S. 10) zu liefern verspricht, konkreten Fallstudien und Praktiken zwar mehr Platz einräumt, sich dabei aber manchmal allzu weit von der begrifflichen oder typologischen Reflexion auf die Formen kollektiver Organisation und auf ihre politische Bedeutung enthält.

8 Beide Bücher ergänzen sich mithin ausgesprochen gut und verschaffen dem Leser einen soliden Überblick über den aktuellen Forschungsstand. Bedauerlicherweise setzen sie allerdings voraus, dass man mit der Chronologie jener Gesetze vertraut ist, die Berufsvereinigungen und Gewerkschaften zunächst unterdrückten und ihnen dann eine Form gaben, womit die rechtlichen Aspekte des Themas doch ein Stück weit vernachlässigt werden. ${ }^{7}$ Ein weiteres Manko der beiden Werke ist sicherlich, dass sie immer wieder ausländische Erfahrungen als Vergleich heranziehen und die Übernahme von Modellen nahelegen, obwohl sie vermeiden möchten, das »französische Modell« zu karikieren oder gar anzuprangern. Da sie aber zu den erwähnten Ländern kaum genauere Angaben machen, nicht einmal bibliographischer Art, erwecken sie manchmal den Eindruck, Staat und Zivilgesellschaft harmonierten außer in Frankreich überall auf natürliche Art und Weise oder, anders gesagt, die Interessenkonflikte ließen sich andernorts problemlos beilegen. Doch sobald wir dem Glauben an den französischen Sonderweg einmal abgeschworen haben, eröffnet sich ein weites Feld für vergleichende Forschungen - die auch der Frage nach dem Export des französischen Modells nachgehen müssten: $\mathrm{Zu}$ denken wäre etwa an die Handelskammern und Handelsgerichte, die Ende des 19. Jahrhunderts auch in Mitteleuropa und Lateinamerika einen gewissen Erfolg zu verzeichnen hatten. Sie können folglich nicht nur als das Feigenblatt angesehen werden, mit dem man die Aporien der Demokratie verdeckt, sondern auch als ein institutioneller Vorschlag, der unter bestimmten Umständen durchaus angemessen ist.

9 Womit die beiden Werke der Forschung nun sicherlich einen großen Dienst erweisen, ist, dass sie eine nach wie vor tonangebende Geschichtsschreibung aufs Altenteil befördern, die - von Étienne Martin-Saint-Léon bis Émile Coornaert - Lobeshymnen auf ein monolithisch wahrgenommenes korporatives System anstimmte, und zugleich das von den Medien kolportierte Bild eines französischen Etatismus und Individualismus widerlegen. Mehr noch: Sowohl Rosanvallon als auch mehrere Autoren des 
Sammelbands La France, malade du corporatisme? gehen detailliert auf die Konstruktion des Korporationsmythos ein, sei es als Vorbild oder als Schreckgespenst, sowie auf die Verwendung der Wörter "Korporation", "Korporatismus " oder auch »Vereinigung", die in den 1840er Jahren oder in der Zwischenkriegszeit en vogue waren, ohne sich unbedingt auf konkrete Projekte zu beziehen. $\mathrm{Zu}$ diesen Autoren zählen vor allem Jacqueline Lalouette mit ihrer schönen Studie zum Vokabular des 19. Jahrhunderts ${ }^{8}$ und Jean-Pierre Hirsch, der sich angesichts der ablehnenden Haltung, mit der man in Frankreich traditionell dem Kollektiveigentum begegnet, über die heute so gegensätzlichen Konnotationen der Wörter »Vereinigung ", »Gewerkschaft«, "Korporation" und »Holding" (S.173) Gedanken macht. Auf diese Weise treten »doppeldeutige Übereinstimmungen« zutage, die nach Ansicht Rosanvallons häufig ein Motor der historischen Entwicklung sind (S. 265) und die darauf beruhen, dass sich Akteure mit ganz unterschiedlichen Werten und Zielen eines gemeinsamen Vokabulars bedienen. Die Texte des Sammelbands, die sich mit der Zwischenkriegszeit und dem Vichy-Regime auseinandersetzen, lösen die proklamierte Verbindung von Diskursen und Praktiken tendenziell weniger gut ein als die anderen, doch werfen sie ein interessantes Licht auf die Überschneidungen, die sich aus vergleichbaren Zweideutigkeiten ergeben. ${ }^{9}$

10 Parallel dazu werden die Kohärenz, aber auch die Varianten und Adaptionen des »jakobinischen" Diskurses - um den Fallstricken dieses Ausdrucks aus dem Weg zu gehen, spricht Rosanvallon lieber von der "politischen Kultur der Allgemeinheit« differenziert dargestellt. Dieser Diskurs lehnt intermediäre Körperschaften aus einem doppelten Grund ab: Sie gelten ihm gleichermaßen als eine Bedrohung der individuellen Freiheit und der nationalen Einheit. Sowohl zu Zeiten der Französischen Revolution wie um die Wende zum 20. Jahrhundert wurde der religiöse Orden als Archetypus einer solchen Körperschaft wahrgenommen, der für alles stand, was man am korporativen Modell als bedrohlich empfand. Sowohl Rosanvallon als auch Philippe Minard zeichnen die Erstarrung des »korporativen« und des »jakobinischen« Modells im Verlauf jener zwischen 1805 und 1825 geführten Debatten um die Wiedereinführung der Korporationen nach. Minard hebt außerdem hervor, wie sehr das Bild einer "totalen Institution", das im Zuge dieser Debatten von den Korporationen des Ancien Régime entstanden ist, von unserem heutigen Bild der Korporation abweicht. Denn im Wissen um ihre Geschichte und ihre Vielgestaltigkeit begreifen wir sie heute eher als eine »ungleiche und hierarchische Gliederung anerkannter Gruppierungen «. ${ }^{10}$ Auch sein Mitherausgeber Kaplan unterstreicht, dass die Korporation unter dem VichyRegime zur "Metonymie einer Reihe positiver Eigenschaften und Tugenden [wurde], die man mit der langen Epoche des Ancien Régime assoziierte und die bis in ein Mittelalter zurückreichen sollte, von dem man nur eine vage Vorstellung hatte ${ }^{11}{ }^{11}$ In einem der anregendsten Beiträge erinnert Clare Crowston daran, wie sich am Ende des 19. Jahrhunderts ein Diskurs über die alten Korporationen entwickelte, der nur sehr wenig mit den Sichtweisen heutiger Historiker zu tun hat. Im Gegensatz zur Gilde der Schneiderinnen aus dem 18. Jahrhundert, einer rein weiblichen, sehr hierarchischen und offiziellen Organisation, versteht sich die 1892 von sozial engagierten Katholiken gegründete "Union de l'Aiguille« als "gemischt" (das heißt, sie setzt sich aus Arbeitgeberinnen und Arbeitnehmerinnen zusammen). Der »Bund der Nadel« tritt für die Solidarität zwischen den Klassen ein, misstraut dem Staat und wird faktisch von männlichen Geistlichen und wohltätigen Schirmherrinnen geleitet. Crowston hat sich in diesem Aufsatz ein dreifaches Ziel gesteckt, dem sie absolut gerecht wird: Es gelingt 
ihr, zum einen die Kontinuitätsbehauptungen der Akteure zurückzuweisen, zum anderen die Triebkräfte hinter deren Suche nach einem historischen Bezugspunkt zu erläutern und drittens die Auswirkungen, die dieses »Modell« auf die Akteure hatte, zu verstehen. ${ }^{12}$

Pierre Rosanvallon räumt den vielfältigen Formen von Berufsorganisationen, die es in der Praxis gab, in seiner Darstellung weniger Platz ein. Doch auch er besteht auf dem konstruierten und nicht in Stein gemeißelten Charakter der pro- und anti-korporativen Diskurse, deren Evolution er durch das 19. Jahrhundert hindurch verfolgt. Insbesondere den "Jakobinismus « betrachtet er nicht als ein Modell, das 1791 oder 1793 ein für allemal starr formuliert worden wäre. Stattdessen legt er Wert darauf, dass Thiers und Guizot eine regelrechte Neufassung dieser Lehre eingeleitet und damit »der Allgemeinheit neue Kleider« angelegt hätten. Ab der zweiten Hälfte des 19. Jahrhunderts seien die intermediären Körperschaften aus verwaltungstechnischen Gründen in begrenztem Umfang als "Krücken« der Administration und später als Schutzschilde gegen den Sozialismus akzeptiert worden (der wiederum als Folge eines übersteigerten Individualismus galt). Die Legalisierung der Gewerkschaften und Arbeitgeberverbänden und später der Vereine allgemein sei somit vor einem gleichbleibenden "Horizont « erfolgt (S. 80), einfach als Reaktion auf diverse »Bewährungsproben«. Praktische Erfordernisse, nicht das Ziel einer Selbstregulierung des Sozialen, hätten für mehr Toleranz gegenüber der organisierten Zivilgesellschaft gesorgt. Diese Entwicklungen seien im 20. Jahrhundert durch die Einrichtung eines neuen Hygiene- und Sozialstaats verstärkt worden, der mit seinen Subventionen über neue Instrumente verfügt habe, um die Vereinigungen einzubeziehen. Rosanvallons innovative Theorie entwirft ein Szenario, in dem sich die Zivilgesellschaft mitnichten dem Staat entgegensetzt, sondern sich vielmehr im Zusammenspiel mit ihm auf komplexe Art und Weise herausbildet.

$\mathrm{Zu}$ bedauern ist lediglich, dass diese nützliche Dekonstruktion bei Rosanvallon - wie vereinzelt auch in dem von Kaplan und Minard herausgegebenen Sammelband - mit rätselhaften Formulierungen im Hinblick auf das "Soziale« versetzt ist: So ist hier etwa von seiner »dichten Materialität « ${ }^{13}$ seiner "Verdrängung " und »Unterschlagung" oder gar vom "sensiblen Fleisch" und den »sensiblen Identitätsprinzipien« des Sozialen die Rede. ${ }^{14}$ Solche Formulierungen, die an die Klischees von der Zivilgesellschaft denken lassen, werden aber der Komplexität dieses "Sozialen" nicht gerecht, das nicht unbedingt einheitlich oder frei von inneren Konflikten ist und von dem man zunächst einmal feststellen muss, ob es sich im Gegensatz zum Staat, zur Politik, zum Recht, zum Markt, zum Instrumentellen usw. definiert. Dieselben Zweifel mögen aufkommen, wenn Kaplan und Minard am Ende ihrer Einleitung eine "andere Sozialgeschichte« im Sinne einer Sozialgeschichte der »Kultur der Gegenmächte« (S. 31) fordern. Denn in Wirklichkeit ist die Geschichte, die sie nachzeichnen, wesentlich komplexer und bei weitem nicht so bipolar; mit derartigen Formulierungen werden die Autoren der historischen Komplexität nicht gerecht und erweisen auch ihrem Forschungsprogramm keinen guten Dienst.

\section{Berufsstand, Identität, Institution}

13 So verdeutlichen die beiden Werke, die eine Synthese der jüngsten Arbeiten auf diesem Gebiet darstellen, die Komplexität der Welt kollektiver Organisationen und die 
Notwendigkeit, sie besser zu analysieren. Es geht hier deshalb weniger darum, auf Defizite als auf komplementäre Perspektiven zu verweisen.

Ein erstes Problem ergibt sich aus dem Umstand, dass sich die Autorinnen und Autoren von La France, malade du corporatisme? oft auf (der Politikwissenschaft entlehnte oder eigenständige) Modelle oder Idealtypen von Korporationen beziehen, ohne dass ihnen deshalb eine gemeinsame Typologie zur Verfügung stünde. Für künftige Forschungen wird es in der Tat entscheidend sein, die unterschiedlichen Funktionen von Gewerkschaften oder Vereinigungen anzuerkennen und ein nachvollziehbares Modell auf der Basis von Fallstudien zu entwickeln. Wenn man es damit ernst meint, muss man zumal zu einer Konzeption der kollektiven Organisationen Stellung nehmen, die diese als ökonomische, auf eine Reihe von Bedürfnissen reagierende Institutionen betrachtet. Ob man diese Bedürfnisse nun im Sinne von »Transaktionskosten« beschreiben möchte oder nicht, auf jeden Fall geht es bei ihnen um Informationsvermittlung, Koordination, Sanktionierung von Betrug, Verbreitung von Fachwissen (insbesondere durch die Berufsausbildung), genossenschaftliche Hilfe auf Gegenseitigkeit und Lobbyismus. ${ }^{15}$ In dieser Hinsicht unterschiede sich die Berufsorganisation (der Arbeitgeber, Arbeiter oder anderer) von anderen Vereinigungsformen und verdiente eine eigene Untersuchung. Die sogenannten Institutionalisten unter den ökonomen haben diesen Institutionen, die weder dem Staat noch dem Unternehmen zuzurechnen sind, nicht oft ihre Aufmerksamkeit gewidmet; und doch wären einige ihrer Ansätze es durchaus wert, auf der Grundlage konkreter historischer Studien diskutiert zu werden. ${ }^{16}$ Denn mit ihrer Hilfe ließe sich vielleicht genauer bestimmen, was Gewerkschaften oder Verbände tun, in welchem Maße man sie als Dienstleister betrachten kann und in welcher Hinsicht ihre soziale Rolle diese Funktion gegebenenfalls übersteigt. Das komplexe Zusammenspiel zwischen öffentlichen und unternehmensbezogenen Dienstleistungen, die von Unternehmen, dem Staat oder kollektiven Organisationen mit unterschiedlichem Status erbracht werden, könnte in diesem Lichte ebenso untersucht werden wie das Verhältnis zwischen Funktionen der Geselligkeit, der Kooperation und der Ausgestaltung des Kollektivs. ${ }^{17}$

Somit könnte man sich von der Methode, nach der Francine Soubiran-Paillet und Marie-Lys Pottier arbeiten, dazu anregen lassen, eine von den Satzungen ausgehende Typologie der Gewerkschaften und Unternehmerverbände zu erstellen: Diese Methode ist zugleich flexibel und systematisch, und sie erlaubt es, eine breite Palette von Funktionen in Betracht zu ziehen. ${ }^{18}$ Es wäre zweifellos wünschenswert, wenn neben solchen allgemeineren Untersuchungen auch mehr Arbeiten zur Verfügung stünden, die sich wie einige Aufsätze in La France, malade du corporatisme? oder wie manche Dissertationen auf eine einzige Branche konzentrieren ${ }^{19}$, ohne sich dabei auf diejenigen Berufe zu beschränken, die sich besonders frühzeitig und besonders gut sichtbar organisierten. So ließe sich auch verstehen, was eventuell der Gründung oder dem Fortbestand einer kollektiven Organisation entgegenstand. Man sollte die Gründung einer bestimmten Organisationsform möglichst nicht mechanisch von bestehenden Bedürfnissen ableiten, sondern vielmehr die widerstreitenden Strategien der Akteure (in den unterschiedlichen Berufssparten, in mit ihnen verbundenen Berufen, in der örtlichen oder nationalen Verwaltung ...) in Rechnung stellen, so wie es Philippe Minard vorgeschlagen hat (S. 44).

Der Nachdruck, der von Wirtschaftswissenschaftlern auf den Begriff der Durchsetzung und auf die Vorstellung der ihre Normen selbst durchsetzenden Institutionen gelegt wird, 
wirft darüber hinaus die Frage nach der Rolle auf, die kollektive Institutionen bei der Normsetzung und der Normanwendung spielen, und nach ihrer Wechselwirkung mit den Gerichten - eine Frage, die für die Historiographie leider bislang nicht von großem Interesse gewesen ist. Einerseits stimmt es, dass man Recht, Ökonomie und Geschichte nur schwer zusammendenken kann, andererseits zeigen die immer provokativen, aber überaus fruchtbaren Ansätze, die Alain Cottereau auf Grundlage seiner Studie über die Conseils de prud'hommes ${ }^{a}$ entwickelt hat, dass es sehr wohl Quellen gibt, die anhand einer Untersuchung von Streitsachen nachvollziehbar machen, wie die kollektive Produktion der - orts- oder berufsspezifischen - „Verkehrssitten« ablief, die als Konsens oder Streitgegenstand den gemeinsamen Bezugspunkt der Interessengruppen bildeten. ${ }^{20} \mathrm{Nur}$ auf der Basis einer solchen Untersuchung können wir beurteilen, ob kollektive Organisationen über eine "Selbstregulierung« verfügen oder nicht - wobei sie oft auch Einfluss auf Regelungen nehmen, die nicht ausschließlich ihre eigenen Mitglieder betreffen. Man muss nicht nur die tatsächliche Praxis der Korporation, sondern parallel auch die des Gesetzes berücksichtigen, die nicht dem abstrakten Bild entspricht, das die "politische Kultur der Allgemeinheit" von ihr entwirft: Denn diese tatsächliche Praxis des Gesetzes lässt es durchaus zu, dass Sachverständige, die von den Institutionen des Berufsstands ausgewählt worden sein können, aber nicht müssen, als Schiedsrichter und Experten, ja sogar als Richter auftreten.

Unter den Funktionen der Korporationen, Gewerkschaften und Verbände darf man deshalb die Beilegung von Rechtsstreitigkeiten zwischen Mitgliedern oder mit Außenstehenden nicht vernachlässigen; dasselbe gilt für ihr Verhältnis zu jenen sehr speziellen Formen der Rechtsprechung wie den Conseils de prud'hommes oder den Handelsgerichten. Doch ihre Funktionen sind natürlich vielfältiger; sie beinhalten Dienstleistungen für den Staat (Klassifikation, Kontrolle, Information) sowie für die eigenen Mitglieder oder andere kollektive Organisationen: Es ist in der Tat ein regelrechtes System, das da Ende des 19. Jahrhunderts entstanden ist und das mehr oder weniger öffentliche lokale Organisationen umfasst wie Handelskammern, Oberste Räte [conseils supérieurs], Gewerkschaften, berufsübergreifende Gewerkschaftskartelle (auf deren Besonderheit Rosanvallon im 10. Kapitel seines Buchs überzeugend eingeht), parlamentarische Kommissionen, gemeinnützige oder andere Vereine, ganz zu schweigen von den internationalen Organisationen und später von der Wirtschaftsplanung. Hat man einmal die Unangemessenheit einer Konzeption erkannt, die den Staat und den Einzelnen einander unvermittelt gegenüberstellt, gilt es, dieses zwischen Konkurrenz, Allianz und wechselnder Rollenverteilung changierende Verhältnis zwischen den »intermediären Körperschaften « zu verstehen. ${ }^{21}$

Da vor dem Hintergrund unserer Überlegungen der Aktionsradius einer Gewerkschaft oder einer Berufsvereinigung alles andere als gegeben zu sein scheint, muss man auch im Hinblick auf die Beziehungen zwischen kollektiver Organisation und »Berufsstand « weiter gehen als bisher. Wie die Autor(inn)en des Bandes La France, malade du corporatisme? kommt auch Rosanvallon in diesem Zusammenhang auf die Arbeiten William $\mathrm{H}$. Sewells zurück, um dessen Schlussfolgerungen allerdings stark zu relativieren..$^{22}$ Die Bedeutung, die sie der Unschärfe des korporativen Vokabulars und der Mythenbildung beimessen, führt in der Tat zu einer Relativierung der von Sewell beschriebenen Kontinuitäten. Um die Beziehungen zwischen Berufsstand und Organisation aber aufklären zu können, wäre ein eigenes Forschungsprogramm erforderlich: Einerseits lässt sich die Korporation unter anderem »durch eine gemeinsame, normalerweise berufliche Tätigkeit« definieren, ${ }^{23}$ andererseits ist auch 
die Definition der Berufsstände oder Berufe selbst fließend und wird von den formalen Organisationen beeinflusst. Sie kommt nicht unbedingt in einer Identität zum Ausdruck; außerdem sind die Maßstäbe, die für die Identitätsbildung eine Rolle spielen mögen, nicht unbedingt dieselben, die Wirtschaftsakteure oder der Staat als relevant für die Schaffung einer passenden Organisation betrachten; einer Organisation, die Gewicht haben, Statistiken zur Verfügung stellen oder auch den Rahmen für die Wahl von Vertretern abgeben kann. ${ }^{24} \mathrm{Zu}$ diesen praktischen Fragen kommen Unsicherheiten, Konflikte und Abwägungen hinzu, die die Identitäten selbst (und die Interessen) tangieren: Auch wenn man sich zugleich mit einem Berufsstand, einer Branche, einer Stadt, einer Region, einem Unternehmen und einer sozialen Klasse identifizieren kann, wird die Organisation natürlich einem dieser Aspekte den Vorrang geben. Insbesondere die Verbindung von Beruf und Ort ist alles andere als selbstverständlich, außer im Falle bestimmter, auf eine Stadt konzentrierter Industrien. ${ }^{25}$

Diese Fragen wirft Michel Pigenet mit Blick auf die Hafenarbeiter auf, die er mit einer ausgezeichneten, gleichermaßen auf historische wie auf soziologische Arbeiten gestützten Studie bedacht hat: Er zeigt die Verbindungen zwischen den Organisationsformen und den konkreten Eigenarten dieses Gewerbes, das an der Schnittstelle von Transportwesen und Handel situiert ist, wobei Pigenet stärker dessen Verbindung zum Hafen als die zur Stadt oder zu einem Unternehmen hervorhebt. ${ }^{26}$ Obwohl auch Yannick Marec anregende Fragen zu berufsübergreifenden Organisationen und den Gründen stellt, warum sich manche Berufsgruppen mit den Angestellten zu Verbänden zusammenschließen, und Clare Crowston betont, dass die Glaubwürdigkeit des korporativen Diskurses der Union de l'Aiguille nicht zuletzt an einer Branche hängt, in der Arbeitgeberinnen großes Gewicht haben, so gehen doch sonst wenige Autoren der Anthologie dieser Frage des Berufsstands und seiner komplexen Verbindungen zur Organisation nach. Auch Rosanvallon beschäftigt sich nicht weiter damit. Die »berufsständische Idee« scheint also manchmal auf einen recht vagen Vorrang des Ökonomischen und des Sozialen vor der Politik hinauszulaufen, eine Unklarheit, die vielleicht dem Tun der Akteure selbst geschuldet ist. Doch die Spezifizität von Formen wie der Gewerbemonographie des nationalen Arbeitsamtes ${ }^{27}$ oder auch der Berufsanerkennung von Seiten des Staates (Diplome, Zugangshürden), die selbst wiederum eine Geschichte hat, könnten den Anstoß zu weiteren Forschungen bilden. Nun gibt es zwar eine Teildisziplin namens Berufssoziologie ${ }^{28}$, doch ist diese oft ahistorisch und setzt die zeitgenössischen Formen der Anerkennung einfach voraus: Ein Dialog mit den Historikern des Korporatismus wäre hier zweifellos besonders lohnenswert. Würde man auf diese Weise das Nachdenken über die Stellung des Gewerbes oder Berufs in der Geschichte des Korporatismus vorantreiben und betonen, dass Interessen und Identitäten hier nie von vornherein gegeben sind, dann könnte es vielleicht endlich gelingen, sich von jedem idyllischen oder "natürlichen« Bild der Zivilgesellschaft zu verabschieden.

\section{Geschichte der Zivilgesellschaft und Geschichte des Staates}

20 Durch ihre differenzierte Darstellung regen beide Werke zu einer weitergehenden Erforschung der Struktur und inneren Konflikte der Zivilgesellschaft an; man könnte dasselbe über den Staat oder die Verwaltung sagen, wie auch immer man sie definiert. 
Auch wenn der Staat und seine Verwaltung nicht explizit Gegenstand dieser Studien sind, die sich eher für die Schnittstelle zwischen Zivilgesellschaft und Staat bzw. für die autonomen Organe ersterer interessieren, taucht das Thema doch auf und macht einen Dialog mit einer Staatsgeschichte, die sich selbst mitten im Umbruch befindet, wünschenswert. ${ }^{29}$

21 So erwähnen beide Bände die Bedeutung der Blüte der Conseils Supérieurs am Ende des 19. Jahrhunderts (vor allem des Conseil Supérieur du Travail), die bei den Ministerien angesiedelt waren und durch das Auswahlverfahren für ihre Mitglieder eine Umstrukturierung der Basiseinheiten der kollektiven Organisation mit sich brachten. Auch wenn die vorliegenden Untersuchungen vergleichsweise schnell über die Debatten um Projekte für ein »Wirtschaftsparlament» hinweggehen, wird doch verschiedentlich auf die Tatsache hingewiesen, dass der Staat durch die Initiierung oder Anerkennung von Gewerkschaften oder Vereinigungen nicht nur nach Vermittlern suchte (etwa nach pädagogischen Vermittlern, wie es Rosanvallon im Hinblick auf die Ligue de l'Enseignement hervorgehoben hat), sondern auch nach Ratgebern, Experten, Informanten, sogar Organisatoren. Die Organisationsmenschen aber, diejenigen, die einen Großteil ihrer Zeit ehrenamtlich oder bezahlt in diesen Strukturen verbringen, um sie zu schaffen oder funktionstüchtig zu halten, sind in den besprochenen Werken weitgehend abwesend - Steven L. Kaplan, der an den Versuch erinnert, in dem speziellen Kontext von Vichy ein »Verwaltungspersonal des Handwerks" auszubilden (S. 435), stellt dabei eine interessante Ausnahme dar. Hier haben wir es mit einem Gebiet zu tun, das zahlreiche Anknüpfungspunkte für zukünftige Forschungen bietet, die man sich etwa in Gestalt von Prosopographien oder aber von realitätsnahen Analysen der Identitäten, Arbeitsweisen und Mittlerrollen vorstellen könnte, die Aktivisten, Funktionäre und auch die Beschäftigten kollektiver Organisationen oft bei der Verbreitung von Ideen, Wörtern oder statistischen Kategorien einnahmen. ${ }^{30}$

22 Abgesehen von dieser Geschichte der Verwaltung von Organisationen bzw. der Organisationen in ihren Verbindungen mit der Verwaltung regen beide Bände auch zum Nachdenken über die Staatsgeschichte im engeren Sinne an. In La France, malade du corporatisme? geschieht dies eher auf indirektem Wege, da die Staatsgeschichte hier nicht unmittelbar Thema ist, in der Einleitung jedoch der besonderen Rolle der Verwaltung des Wirtschaftsplans als Ortes der Annäherung und Konsultation der "gesellschaftlichen Kräfte" nach 1945 gedacht wird: Es wäre überaus wünschenswert, diese Episode im Rahmen einer Langzeitbetrachtung der Entwicklung von Formen der Beratung eingehender zu studieren. Jean-Pierre Le Crom weist auch auf das wichtige Problem der Koordination der Regierungsarbeit, der Kompetenzen und Mittel der unterschiedlichen Ministerien hin (S.372): Damit berührt er die Frage des "Korporatismus« jeder Administration, die sich womöglich mit den Interessen einer Branche identifiziert, ebenso wie den Aspekt, dass es Administrationen durch ihr Zurückgreifen auf intermediäre Körperschaften gelingen kann, Steuerungsaufgaben zu lösen, ohne den Umfang des öffentlichen Dienstes zu vergrößern.

Pierre Rosanvallon behauptet ausdrücklich, dass die Form von Anerkennung, die kollektive Organisationen erfuhren, weitgehend von einem »Imperativ der Regierbarkeit« abhing - von den Diensten, die sie für eine Verwaltung erbringen konnten, die weniger reich, weniger gut informiert und weniger effizient war, als man oft denkt. Diese wegweisende Hypothese stellt die allzu einfachen Grenzen zwischen 
öffentlich und privat in Frage, auch wenn im Hinblick auf einige Schlüsse, die Rosanvallon aus ihr ableitet, durchaus noch Diskussionsbedarf besteht. Denn einerseits beschreibt er eine Umorganisation von oben, eine "Managementlösung [remède managérial] für die Defizite der atomisierten Gesellschaft« (S. 216). Damit aber vergisst er, dass es niemals gelungen ist, eine allgemeine Rekorporatisierung zu verordnen, wie es sowohl die Studien über Vichy in La France, malade du corporatisme? als auch die Kontroversen der 1820er Jahre zeigen: Immer wieder nämlich musste mit der unregelmäßigen, spontanen Organisation der Berufsstände, dem Problem der Definition ihrer Grenzen oder aber dem Problem der Finanzierung der Organisationen gerechnet werden. Das Scheitern dieser Ansätze, mit allem, was es über die Erwartungen des Staates und die von einer "gelenkten « Organisation aufgeworfenen praktischen Probleme aussagt, wurde möglicherweise noch nicht ausreichend beleuchtet.

Um die Behauptung zu stützen, dass die Vereinigungen in Frankreich nur als ein Ausdruck der Vereinigungsfreiheit oder aus instrumentellen Gründen, nicht aber als politische Institutionen im vollen Sinne anerkannt wurden, überzeichnet Rosanvallon mitunter die Grenzen zwischen Verwaltung und Politik, womit er auf eine zweifellos unzureichende Definition dieser Begriffe hinweist, insbesondere für das 19. Jahrhundert. Indem er die Frage der Dezentralisierung mit der Frage der Korporationen in Verbindung bringt, wie es die Akteure der damaligen Zeit taten, behauptet er, dass die Kommune als rein administrative Instanz angesehen wurde, womit er sie auf eine Stufe mit der "Zivilgesellschaft", ja sogar der Familie stellt; andererseits betont er den besonderen Status, den sie in Paris besaß, wo jede Wahl zwangsläufig politisch sein musste. Mit Verweis auf die Rolle, die die »Höchststeuerzahler« [les »plus imposés«] lange Zeit neben den Stadträten spielten, geht er soweit zu behaupten, dass »die Kommune eine Art von >Interessenskonsortium< und keine politische Gemeinschaft war" (S. 365). ${ }^{31}$ Auch wenn dieser Ansatz nicht uninteressant ist, wirft eine solche Formel doch die Frage auf, was dann, wenn so viele Funktionen in die Rubrik der Verwaltung oder des wirtschaftlichen Interesses verwiesen werden, im eigentlichen Sinne überhaupt noch politisch ist. Man hat fast den Eindruck, Rosanvallon nähere sich mit seiner Vision einer ungreifbaren reinen Politik, die mit dem Parlament identisch zu sein scheint, einem quasi jakobinischen Gesetzeszentrismus an. An anderer Stelle aber weist er durchaus darauf hin, dass etwa die Grenzen zwischen Staat und Notabeln (S. 430) schwer zu ziehen sind. Vieles spricht also dafür, dass man weniger von unüberbrückbaren Gräben zwischen »Verwaltung" und »Politik«, zwischen lokalen und nationalen Normen oder zwischen Zivilgesellschaft und Staat ausgehen sollte, als von kontinuierlichen Übergängen.

$\star \star \star$

»Das französische Modell hat in Wirklichkeit immer mit einer gewissen Distanz zu sich selbst funktioniert, pragmatischer als die zur Schau getragenen Prinzipien erwarten lassen«, resümiert Pierre Rosanvallon (S. 307 f.). So unterschiedlich der theoretische Ausgangspunkt beider Bücher ist, kommt doch sowohl Rosanvallons Werk als auch dem Sammelband von Steven L. Kaplan und Philippe Minard das Verdienst zu, sowohl auf diesen Abstand zwischen Diskurs und Praxis hinzuweisen als auch deren wechselseitige Beeinflussung in den Blick zu nehmen - denn die Diskurse, die man keinesfalls einfach 
als scheinheilig oder irrelevant abstempeln darf, tragen auch dazu bei, den Raum möglicher Praktiken zu modellieren.

Aus diesem Grund gehören beide Werke nicht nur in die Bibliotheken aller Politik-, Sozial- und Wirtschaftshistoriker, die sich mit den letzten zwei Jahrhunderten befassen, sondern auch in die Bibliothek eines jeden Bürgers, der sich mit bestimmten journalistischen Verkürzungen nicht abfinden möchte, etwa im Hinblick auf den französischen Sonderweg, Tocqueville oder den "Korporatismus« der Beamten. Jedes dieser Bücher, die beide ihre Stärken und Schwächen haben, bietet eine Teilansicht. Sie machen aber zumal deutlich, dass mittlerweile eine kritische Masse an historischer Forschung über die »Zivilgesellschaft« erreicht ist und dass es an der Zeit wäre, die differenzierten Ergebnisse dieser Forschung der Öffentlichkeit zugänglich zu machen.

Aus der Summe beider Bücher gehen der »jakobinische« und der »korporative« Mythos ziemlich zurechtgestutzt hervor, während sich zugleich ein Forschungsfeld abzeichnet, das erst noch zu vermessen ist - und das noch keinen Namen hat. Da man den Begriff der »Zivilgesellschaft « heute in Frankreich tendenziell mit der mythischen Vorstellung eines von den Unreinheiten des politischen Betriebs freien "Sozialen« in Verbindung bringt und der Begriff der "intermediären Körperschaften" doch offenbar einem organizistischen Bild der Gesellschaft verhaftet ist, das von den zahlreichen Funktionen kollektiver Organisationen nur deren Rolle als "Mittler « zwischen dem Einzelnen und Staat hervorhebt, scheinen beide gewisse Probleme und Einschränkungen mit sich zu bringen. Doch diese begrifflichen Schwierigkeiten sollten Historiker nicht daran hindern, sich - idealerweise im Zusammenspiel mit Vertretern der Institutionenökonomik und Berufssoziologen - über die Gewerkschaften, die Vereinigungen und die vielzähligen Strukturen $\mathrm{zu}$ beugen, die sie zusammengruppieren, der Verwaltung entgegensetzen oder mit ihr verbinden. Dabei sollten diese kollektiven Organisationen sowohl für sich genommen untersucht werden, in ihren internen Konflikten, als auch mit Blick darauf, was wir in Bezug auf die Geschichte der Gewerbe und Berufe und die Geschichte des Staates aus ihnen lernen können.

\section{BIBLIOGRAPHIE}

Abbott, A. (1988): The System of Professions. An Essay on the Division of Expert Labor, Chicago: University of Chicago Press.

Andrieu, C. / Le Beguec, G. / Tartakowsky, D. (Hg.) (2001): Associations et champ politique, la loi de 1901 à l'épreuve du siècle, Paris: Publications de la Sorbonne.

Aoki, M. (2001): Toward a Comparative Institutional Analysis, Cambridge, Mass.: MIT Press.

Baruch, M.-O. / Duclert, V. (Hg.) (2000): Serviteurs de l'État, une histoire politique de l'administration française 1875-1945, Paris: La Découverte.

Bryant, C. G. A. (1993): »Social Self-Organisation, Civility and Sociology. A Comment on Kumar's `Civil Society/«, British Journal of Sociology, Bd. 44, Nr. 3, S. 397-401. 
Bryant, C. G. A. (1994): »A Further Comment on Kumar's `Civil Society««, British Journal of Sociology, Bd. 45, Nr. 3, S. 497-499.

Cerutti, S. (1990): La ville et les métiers. Naissance d'un langage corporatif(Turin, XVIIe-XVIIIe siècle), Paris: Éditions de l'EHESS.

Chatriot, A. (2002): La démocratie sociale à la française. L'expérience du Conseil national économique, 1924-1940, Paris: La Découverte.

Chatriot, A. / Lemercier, C. (2002): »Les corps intermédiaires«, in: Duglert, V. / Prochasson, C. (Hg.): Dictionnaire critique de la République, Paris: Flammarion, S. 691-698.

Chatriot, A. / Lemercier, C. (2008): »Une histoire des pratiques consultatives de l'État«, in: Offerlé, M. / Rousso, H. (Hg.): La fabrique interdisciplinaire. Histoire et science politique, Rennes: Presses universitaires de Rennes, S. 191-203.

Cottereau, A. (Hg.) (1987): »Les prud'hommes, XIXe-XXe siècles«, Mouvement Social, Okt.-Dez., Nr. 141.

Cottereau, A. (2004): "La désincorporation des métiers et leur transformation en >publics intermédiaires : Lyon et Elbeuf, 1790-1815«, in: Kaplan / Minard (Hg.) (2004), S. 97-145.

Crowston, C. (2004): »Du corps des couturières à l'Union de l'Aiguille. Les continuités imaginaires d'un corporatisme au féminine«, in: Kaplan / Minard (Hg.) (2004), S. 197-232.

Descimon, R. / Guéry, A. (1989): „Un État des temps modernes?«, in: Bourgière, A. / Revel, J. (Hg.): Histoire de la France, 2. Bd.: L'État et les pouvoirs, Paris: Seuil, S. 181-356.

Descimon, R. / Schaub, J.-F. / Vincent, B. (Hg.) (1997): Les figures de l'administrateur. Institutions, réseaux, pouvoirs en Espagne, en France et au Portugal, XVIe-XIXe siècle, Paris: Éditions de l'EHESS.

Dubar, C. / Tripier, P. (2003 [1998]): Sociologie des professions, Paris: Armand Colin.

Dumons, B. (2000): »Pouvoir municipaux et élites administratives des villes du Sud-Est de la France (1884-1940). Historiographie, méthodes, perspectives«, Bulletin de la SHMC, Nr. 3/4, S. 145155.

Fraboulet, D. (2007): Quand les patrons s'organisent. Stratégies et pratiques de l'Union des industries métallurgiques et minières 1901-1950, Villeneuve d'Ascq: Presses universitaires du Septentrion. Gould, R. V. (1995): Insurgent Identities. Class, Community and Protest in Paris from 1848 to the Commune, Chicago/London: University of Chicago Press.

Greif, A. (1998): »Théorie des jeux et analyse historique des institutions. Les institutions historiques du Moyen Âge«, Annales HSS, Bd. 53, Nr. 3, Mai/Juni, S. 597-633.

Hall, J. / Trentmann, F. (Hg.) (2005): Civil Society. A Reader in History, Theory and Global Politics, London: Palgrave.

Harris, J. (Hg.) (2003): Civil Society in British History. Ideas, Identities, Institutions, New York: Oxford University Press.

Hirsch, J.-P. (1991): Les deux rêves du commerce. Entreprise et institution dans la région lilloise (17801860), Paris: Éditions de l'EHESS.

Hirsch, J.-P. (2004): »L'impossible propriété collective«, in: Kaplan / Minard (Hg.) (2004), S. 171194.

Hirsch, J.-P. / Minard, P. (1998): » Laissez-nous faire et protégez-nous beaucoup . Pour une histoire des pratiques institutionnelles dans l'industrie française (XVIIIe-XIXe siècle)«, in: 
Bergeron, L. / Bourdelais, P. (Hg.): La France n'est-elle pas douée pour l'industrie?, Paris: Belin, S. 135158.

Hunt, L. / Sheridan, G. (1986): „Corporatism, Association, and the Language of Labor in France, 1750-1850«, Journal of Modern History, Bd. 58, Nr. 4, S. 813-844.

Joly, H. (Hg.) (2004): Les comités d'organisation et l'économie dirigée du régime de Vichy, Caen: Centre de recherche d'histoire quantitative.

Kaplan, S. L. (2001): La fin des corporations, Paris: Fayard.

Kaplan, S. L. (2004): »Un creuset de l'expérience corporatiste sous Vichy. L'Institut d'études corporatives et sociales de M. Bouvier-Ajam«, in: Kaplan / Minard (Hg.) (2004).

Kaplan, S. L. / P. Minard (Hg.) (2004): La France, malade du corporatisme? XVIIIe-XXe siècles, Paris: Belin.

Kaplan, S. L. / P. Minard (2004): »Introduction. Le corporatisme, idées et pratiques. Les enjeux d'un débat incessant«, in: ebd.

Kieffer, M. (1986): »Aux origines de la législation du travail en France. La légalisation des syndicats et la démocratisation des conseils de prud'hommes«, Dissertation unter der Leitung von Madeleine Rebérioux an der Universität Paris 8.

Kumar, K. (1993): „Civil Society. An Inquiry into the Usefulness of a Historical Term«, British Journal of Sociology, Bd. 44, Nr. 3, S. 375-395.

Kumar, K. (1994): „Civil Society Again. A Reply to Christopher Bryant's ıSocial Self-Organisation, Civility and Sociology «, British Journal of Sociology, Bd. 45, Nr. 1, S. 127-131.

Lalouette, J. (2004): »Les insaisissables corporations du premier XIXe siècle. Enquête sur les usages d'un mot «, in: Kaplan / Minard (Hg.) (2004), S. 147-170.

Lematte, É. (2004): »Institution et changement institutionnel. Le cas de l'ordre institutionnel corporatif et de son abolition «, Studienarbeit (mémoire de DEA) in Wirtschaftsanalyse und Wirtschaftspolitik, EHESS, eingereicht bei Gilles Postel-Vinay.

Lemercier, C. (2004): »Classer l'industrie parisienne au XIXe siècle«, Nomenclatures et classifications. Approches historiques, enjeux économiques. Actes et communications de l'INRA, Nr. 21, Nov., S. 237-271.

Leteux, S. (2010): Libéralisme et corporatisme chez les bouchers parisiens (1776-1944), 2 Bde., Saarbrücken: Éditions universitaires européennes.

Minard, P. (1998): La fortune du colbertisme. État et industrie dans la France des Lumières, Paris: Fayard.

Minard, P. (2004): »Les corporations en France au XVIIIe siècle. Métiers et institutions«, in: Kaplan / Minard (Hg.) (2004), S. 39-51.

Moret Lespinet, I. (2004): „Vers un `corporatisme« républicain ? Les réformateurs de l'office du travail«, in: Kaplan / Minard (Hg.) (2004), S. 355-367.

Offerlé, M. (Hg.) (2003): La société civile en question, Paris: La Documentation française.

Ozouf, M. (1981): »Fortune et infortunes d'un mot«, Le Débat, Juni, Nr. 13, S. 28-39.

Pigenet, M. (2004): »Les travailleurs de la manutention portuaire ou les métamorphoses du modèle corporatif«, in: Kaplan / Minard (Hg.) (2004), S. 253-278. 
Plessis, A. (Hg.) (1993): Naissance des libertés économiques: liberté du travail et liberté d'entreprendre. Le décret d'Allarde et la loi Le Chapelier, leurs conséquences, 1791- fin XIXe siècle, Paris: Institut d'histoire de l'industrie.

Rosanvallon, P. (1989): »Corporations et corps intermédiares«, Le Débat, Nov.-Dez., Nr. 57, S. 190194.

Rosanvallon, P. (1992): Le sacre du citoyen, histoire du suffrage universel en France, Paris: Gallimard.

Rosanvallon, P. (1998): Le peuple introuvable, histoire de la représentation démocratique en France, Paris: Gallimard.

Rosanvallon, P. (2000): La démocratie inachevée, histoire de la souveraineté du peuple en France, Paris: Gallimard.

Rosanvallon, P. (2004): Le modèle politique français. La société civile contre le jacobinisme de 1789 à nos jours, Paris: Seuil.

Rosanvallon, P. (2011) [2002]: »Für eine Begriffs- und Problemgeschichte des Politischen. Antrittsvorlesung am Collège de France [18. März 2002]«, Mittelweg 36, Bd. 20, Nr. 6, S. 43-65.

Sapiro, G. (2004): »Entre individualisme et corporatisme. Les écrivains dans la première moitié du XXe siècle«, in: Kaplan / Minard (Hg.) (2004), S. 279-314.

Schaub, J.-F. (1996): „Le temps et l'État. Vers un nouveau régime historiographique de l'Ancien Régime français«, Quaderni fiorentini per una storia del pensiero giuridico moderno, Nr. 25, S. 127-181.

Sewell, W. H. (1980): Work and Revolution in France. The Language of Labor from the Old Regime to 1848, Cambridge: Cambridge University Press.

Soubiran-Paillet, F. (1999): L'invention du syndicat (1791-1884). Itinéraire d'une catégorie juridique, Paris: Maison des sciences de l'homme/LGDJ.

Soubiran-Paillet, F. / Pottier, M.-L. (1996): De l'usage professionnel à la loi. Les chambres syndicales ouvrières parisiennes de 1867 à 1884, Paris: L'Harmattan.

Topalov, C. (Hg.) (1999): Laboratoires du nouveau siècle, Paris: Éditions de l’EHESS.

Vernus, P. (Hg.) (2002): Les organisations patronales. Une approche locale (XIXe-XXe siècles), Cahiers du Centre Pierre Léon d'histoire économique et sociale, Nr. 1, Sept., Université Lumières-Lyon II.

\section{NOTES}

1. Unter den wegweisenden Arbeiten zu dieser Thematik lassen sich zwei Traditionslinien unterscheiden: Die eine kommt mit Ozouf (1981) und Rosanvallon (1989) aus einer eher politischen Geschichtsschreibung; die andere beschäftigt sich mit Formen wirtschaftlicher Regulierung; vgl. Cottereau (1987), Hirsch (1991), Hirsch / Minard (1998), Minard (1998), Kaplan (2001). Die beiden hier analysierten Werke zeugen in gewissem Maß von der anhaltenden Trennung zwischen diesen beiden Forschungsrichtungen. Auf zwei weitere Sammelbände, die unter anderem wertvolle Editionen von Gesetzestexten enthalten, sei an dieser Stelle verwiesen: Plessis (1993) sowie Andrieu et al. (2001).

2. Kaplan / Minard (2004), S. 27.

3. Hirsch (2004).

4. Zu seiner Geschichte und seinen jüngsten Verwendungen vgl. für Frankreich Offerlé (2003); für andere Länder und hier insbesondere seine Verwendung im Zusammenhang mit der Ablösung des Kommunismus in Osteuropa vgl. einen Austausch im British Journal of Sociology: Kumar (1993); 
Bryant (1993); Kumar (1994); Bryant (1994). Für eine jüngere Bestandsaufnahme der britischen Diskussionen über den Begriff vgl. Harris (2003); sowie Hall / Trentmann (2005).

5. Rosanvallon (2011 [2002]).

6. Rosanvallon (1992), (1998), (2000).

7. Die genausten Untersuchungen dieses Aspekts bieten Kieffer (1986) und Soubiran-Paillet (1999).

8. Lalouette (2004).

9. $\mathrm{Zu}$ den Praktiken finden sich mehr Einzelheiten in einer neueren Publikation: Joly (2004). Bezüglich der Verbindung von Diskursen und Praktiken sei verwiesen auf Chatriot (2002).

10. Vgl. seinen wertvollen Überblick in Minard (2004).

11. Kaplan (2004), S. 445.

12. Crowston (2004).

13. Kaplan / Minard (2004), S. 17 - ein Ausdruck, der mit Bezug auf die Arbeiten von Pierre Rosanvallon gebraucht wird, den sich die Autoren aber zu Eigen zu machen scheinen.

14. Rosanvallon (2004), S. 235 u. 122 ff.

15. Kaplan (2004), S. 76 f., schlägt eine Aufzählung dieser Funktionen vor, die von seinen Mitautoren leider nicht aufgegriffen wird. Für eine Fallstudie und eine allgemeine Reflexion zu dieser Frage vgl. Vernus (2002).

16. Vgl. die Arbeiten von Avner Greif, auf Französisch zusammengefasst in Greif (1998), sowie die von Masahiko Aoki, zusammengefasst in Aoki (2001). Für einen anregenden Vorschlag einer Anwendung auf das hier zur Debatte stehende Gebiet vgl. Lematte (2004). Das Instrumentarium der ökonomischen Analyse wird hier für eine Interpretation der Diskrepanz zwischen Diskursen, Gesetzen und Praktiken genutzt.

17. Um die interessante Typologie von Rosanvallon (Kap. XI) aufzugreifen, der sie allerdings weniger auf die Funktionen einer einzigen Organisation als auf verschiedene Organisationen anwendet.

18. Soubiran-Paillet / Pottier (1996).

19. Namentlich die 2005 unter der Leitung von Jean-Pierre Hirsch fertiggestellte Dissertation von Sylvain Leteux: "Libéralisme et corporatisme chez les bouchers parisiens (1776-1944)«, Universität Lille III; vgl. jetzt Leteux (2010).

a. [Paritätisch besetzte Arbeitsgerichte, die über individuelle Konflikte aus dem Arbeitsvertrag entschieden; A.d.H.].

20. Siehe insbesondere seine Untersuchung Cottereau (2004).

21. Eine vom Centre d'histoire sociale du XXe siècle (CNRS) organisierte Tagung vom 25.-26. November 2004 über »Gewerkschaften und Vereinigungen in Frankreich: Konkurrenz oder Komplementarität?« schlug Fallstudien zu diesen Fragen vor - in Verlängerung der Bemerkungen Yannick Marecs über das Verhältnis zwischen Berufsverein [amicale professionelle] und Gewerkschaft im Fall der Angestellten aus Rouen (Kaplan / Minard [2004], S. 242 f.). Dieses Forschungsprogramm wird verteidigt von Chatriot / Lemercier (2002), (2008).

22. Sewell (1980). Eine interessante Kritik daran hatten bereits Hunt/ Sheridan (1986) vorgetragen.

23. Kaplan / Minard (2004), S. 6.

24. Vgl. Lemercier (2004). $\mathrm{Zu}$ den praktischen Gesichtspunkten der Entscheidung für eine Organisation je nach Standort oder Tätigkeit geben die Arbeiten von Danièle FrabouletRousselier über den Verband der metallverarbeitenden Industrie und des Bergbaus Auskunft; vgl. inzwischen Fraboulet (2007).

25. Bezüglich der Entscheidung zwischen diesen beiden Identitätstypen und ihrer Verbindungen zu formalen Organisationen kann man auf die Ansätze von Cerutti (1990) und Gould (1995) zurückgreifen.

26. Pigenet (2004). 
27. Moret Lespinet (2004).

28. Die im hier behandelten Sammelband lediglich Sapiro (2004) erwähnt. Für einen Überblick vgl. Dubar / Tripier (2003). Abbott (1988) ist eine der Arbeiten dieser Strömung, die der Geschichte am meisten Platz einräumt.

29. Für die Neueste Geschichte vgl. Baruch / Duclert (2000). Diese Erneuerung verdankt sich zumal einem Dialog mit der modernen Geschichte: Vgl. die zusammenfassende Darstellung von Descimon / Guéry (1989); außerdem Schaub (1996). Vgl. auch Descimon / Schaub / Vincent (1997) und Minard (1998).

30. Vgl. die Vorschläge von Topalov (1999), insbesondere die letzten vier Kapitel.

31. Zur jüngeren Historiographie der lokalen Institutionen enthält Dumons (2000) eine sehr umfangreiche Bibliographie.

INDEX

Schlüsselwörter : Frankreich, Jakobinismus, Korporatismus, Zivilgesellschaft

Mots-clés : France, jacobinisme, corporatisme, société civile

\section{AUTEURS}

\section{CLAIRE LEMERCIER}

Claire Lemercier ist Forschungsdirektorin am CNRS (Centre de sociologie des organisations). Nähere Informationen finden Sie hier. 